Proceedings of the 2012 Winter Simulation Conference

C. Laroque, J. Himmelspach, R. Pasupathy, O. Rose, and A. M. Uhrmacher, eds.

\title{
A SIMULATION-BASED DECISION SUPPORT SYSTEM TO MODEL COMPLEX DEMAND DRIVEN HEALTHCARE FACILITIES
}

\author{
Michael Thorwarth \\ Amr Arisha \\ 3S Group, College of Business \\ Dublin Institute of Technology \\ Dublin 2, IRELAND
}

\begin{abstract}
Simulating healthcare processes is a sophisticated endeavor. Treatment processes and patient arrival patterns differ significantly in their statistical attributes and implicate a high degree of variability. In addition, there are several types of interconnected processes of medical staff involved that accompany a patient's journey through the healthcare facility. Replicating this behavior with process flow models in a discrete event simulation model is highly complex and therefore difficult to create while maintaining a high degree of precision.

A framework is thus introduced which delivers an algorithm that allows to implement Multiple Participant Pathway Modeling (MPPM) under the consideration of Flexible Resource Allocation (FRA). This framework is applied on an Irish Emergency Department (ED), the outcome of which is presented here. Results show that scenarios can be investigated which impact several different process flows with a high precision. This provides a solid base for both the interpretation of results and decision making.
\end{abstract}

\section{INTRODUCTION}

Healthcare processes tend to be complex and driven by patients' demand. Many skills and tasks are involved in the process of treating a patient, in addition to the regulations and the procedures that medical staff has to comply with. These accompanying processes, such as documentation, preparation, communication, post processing, etc. add a major proportion of work onto the daily routine of medical staff.

In the context of healthcare, simulation is gaining acceptance among healthcare managements. Querying the database on Harzing (2010), it can be seen that within 15 years the number of studies on Discrete Event Simulation (DES) in EDs has risen exponentially (Thorwarth 2011). In general, simulation in healthcare is used to investigate the current situation in a healthcare facility in order to determine the potential for improvement. Optimization was found to be an integral part of indentifying the right decisions for this healthcare dilemma. Simulation, especially process flow simulation or DES, investigates the flow of patients through a facility in order to apply several improvement measures such as the reduction of waiting time and average length of stay, the optimization of resource utilization, etc.

Jun, Jacobson, and Swisher (1999) and Günal and Pidd (2010) identify DES models that are applied in healthcare. Those models investigating the patient flow within a healthcare facility focus on the movement of the patient through the facility. There, medical staff is treated as a static resource, as examples can be obtained from the following studies: Hoot, LeBlanc, Jones, Levin, Zhou, Gadd, and Aronsky (2008), Yeh and Lin (2007), and Ruohonen, Neittaanmäki, and Teittinen (2006).

Treating medical staff as a static resource is sufficient in cases where the dependency of processes and queues to any potential change of policies is required to be investigated. However, once policies are of interest which take effect on patients and medical staff, further measures have to be taken in order to simulate the workflow within the healthcare facility. An example for such twofold policies would be the 


\section{Thorwarth, and Arisha}

introduction of documentation aids such as speech recognition. The aim would be to reduce the Length of Stay (LoS) of patients and to minimize the documentation effort of staff.

In general practice, the medical staff has got a work list that needs to be processed, while treating a patient of which consists of preparative and concluding tasks. This workflow contains processes that accompany patients and additional off site processes that do not require the presence of the patient.

In order to increase the precision of healthcare process simulation, this work list needs to be combined with the patient pathway. Combining the workflow of medical staff with the patient pathway increases the complexity of the simulation model. Within this research, a simulation framework is proposed where the modeler can incorporate the workflow with the patient pathway with the Multiple Participant Pathway Modeling (MPPM). Another aspect which has to be considered in order to increase the precision of healthcare process simulation is Flexible Resource Allocation (FRA), which is observed in a demand driven environment such as in Emergency Departments (ED). Medical staff is one resource that is often allocated in dependence on the demand of a healthcare facility. This is the case when staff is ordered from a staff pool or a nearby department to help in case of emergency. Capturing such mechanisms is feasible with MPPM and FRA, but the complexity of process flow simulation models is significantly increased, and therefore such models are difficult to design.

In order to propose a solution for this complex issue, a framework is introduced within this paper that allows to automatically generate a grid which can be used in order to replicate healthcare models that incorporate MPPM and FRA. This grid is generally designed to the specifications of the system under investigation, which helps the modeler during the layout phase of the simulation model. The overall aim of the included field study is to increase the precision of simulation models that allow investigation of scenarios and policies that affect both the patient pathway and the workflow of medical staff. An Irish ED was selected to investigate the potential impact of the introduction of a documentation aid.

After having introduced this research, a literature review briefly summarizes the background to the topic. The third section explains the general mechanism of the MPPM and FRA approach, accompanied by the proposed terminology. The modeling of this approach is described in the fourth section by providing a brief layout of the ED, a patient pathway description, Data Mining methodology, and conceptual modeling. Another important aspect of the forth section is that an instruction is provided of how patient flow and workflow is combined by applying the here explained automated modeling. After providing insights into the application of the actual simulation model, the forth section is concluded with a statement on verification and validation results obtained by the comparison of the model with the actual hospital records. Results summarize the contribution of the optimized simulation runs in the fifth section. The last section concludes with a discussion on the further use of this research and it's potential.

\section{LITERATURE REVIEW}

Combining patient pathways with the workflow of medical staff has not yet, to the authors' best knowledge, been investigated in the context of DES in detail. As complexity issues are a potential reason, it is worthwhile to view some aspects of complexity regarding simulation flow models. There are some examples, which are mentioned as they tend to link different processes of several models together, as for example Eatock, Paul, and Serrano (2001) has applied onto two connected models of two interlinked pharmaceutical companies or as Rohleder, Bischak, and Baskin (2007) who has combined two different modeling approaches like DES and System Dynamics into one system. Both examples integrate two models, but they do not link different process types which are connected over one item type within the simulation model.

McCabe (1976) discusses a complexity metric based on graph theory (Berge 1976) and introduces the Cyclomatic metric which describes the behavior of workflow nets. Lassen and van der Aalst (2009) extend the Cyclomatic metric by considering the absolute value of the edges and vertices with the following equation, where the Extended Cyclomatic Metric (ECyM) is a measurement which is used in order to describe the structure of a number of elements of DES models by $E C y M=|E|-|V|+P$ where $E$ represents the number of edges, $V$ the number of vertices and $P$ the strongly connected components. This metric is 


\section{Thorwarth, and Arisha}

applicable to workflow nets which contain "IF THEN" constructs as well as "LOOP"s constructs. Decisions and loops are commonly used within DES models; therefore the Cyclomatic metric is a valid representation. An overview on the complexity degree of assorted models is provided by Thorwarth (2011), where a list is assembled on the basis of information provided on the authors published models.

Modeling the complex behavior of an ED is a challenging task due to the interaction of human and physical resources. Medical staff, for example, is rarely dedicated to one patient or task as they treat several others while waiting for other processes' outcomes. This diversity of process interaction can be described as multitasking, a common feature of ED operations. Yet, multitasking is rarely considered in DES models of EDs (Günal and Pidd 2006). Several tasks and processes, depending on certain resources, result in interrupt-driven system behavior demanding a well adjusted allocation of human and physical resources (Wild, Johnson, and Johnson 2004). The impact of multitasking on ED simulation models certainly warrants further investigation, especially on the background of scarce shared resources. A model that consider utilization under the constraint of multitasking is provided by Thorwarth, Harper, and Arisha (2009).

\section{SPECIFIC APPLIED MECHANISMS}

Two mechanism are described within this section, that allow to capture and represent the dynamics of an $\mathrm{ED}$, where specific and strict working procedures have to be considered by medical staff, even though they are not bound to a certain workstation. The here described mechanisms are not retrieved in the current literature to the best knowledge of the authors.

\subsection{Mechanism A: Flexible Resource Allocation}

Flexible Resource Allocation (FRA) can be observed wherever resources are available on call for different tasks - the resource is not bound to a specific activity or task and flexible located. Multiple Participant Pathway Modeling however, is applicable to systems, where processes of different resources are linked in order to complete a task or finish a product. In brief: FRA describes the sharing of resources among several processes. Thorwarth, Harper, and Arisha (2009) provide a model for the calculation of the utility level of medical staff within an ED where FRA is observed.

\subsection{Mechanism B: Multi Participant Pathway Modeling}

In order to illustrate the FRA and MPPM mechanisms and deliver an understanding of its complexity, a small model Figure (1) is first discussed: Within the model two succeeding processes are sequentially aligned describing the patient pathways in an abstracted setting which share the same staff, and here it is described as the passive pathway. The treated patients enter this pathway and are receiving further treatment according to the laid out pathway, first process $a$, then process $b$. A staff resource (following the active pathway) is joining the patient by providing the desired treatment, assigned as process $a$, once the treatment is concluded the member of staff moves back to the pool, performing whichever duties are necessary there, while the patient proceeds to the next process step $b$. A full circle describing the preparation, treatment and concluding processes is labeled here as active pathway because the staff resource is actively contributing to the outcome of the passive pathway. Adding prior and succeeding tasks to the patient treatment processes $a$ and $b$ are described as Multiple Participant Pathway Modeling (MPPM). Those added tasks may consist of preparation and documentation.

\section{MODELING}

Including FRA and MPPM in simulation modeling follows the same methodology as the modeling procedure proposed by Banks, Carson II, Nelson, and Nicol (2005), with one main consideration: before the model is translated into an executable model, the modeler should consider the automated modeling described in 


\section{Thorwarth, and Arisha}

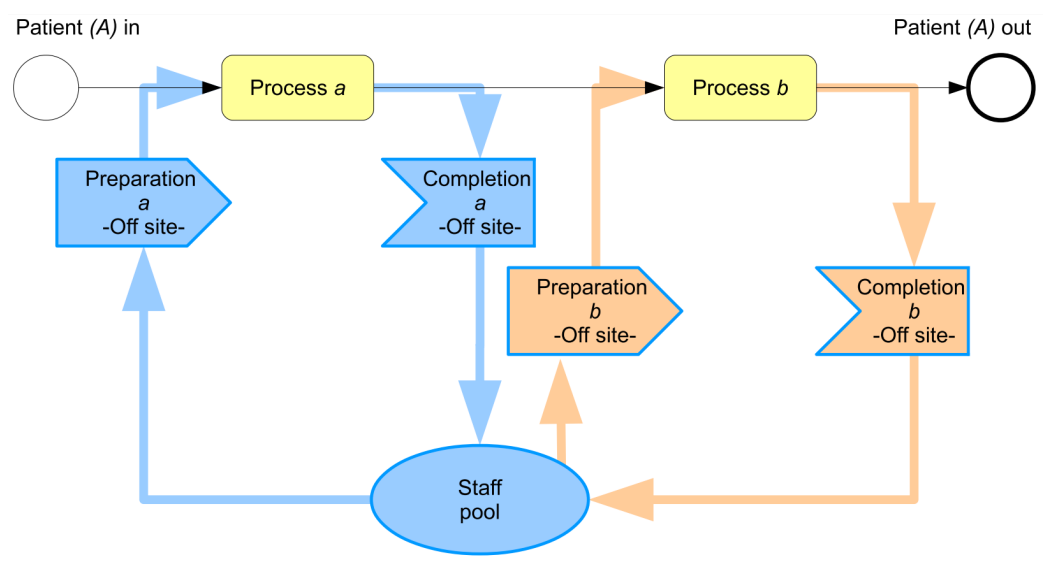

Figure 1: Illustration of the mechanism behind Multiple Participant Pathway Modeling (MPPM) under the consideration of Flexible Allocated Resources (FRA).

Section 4.6. In order to apply automated modeling the simulation package has to support a programming interface which allows to automatically deflate a process grid network out of elements that are previously designed and thus can be used as a template for hierarchical modules.

In addition to the above mentioned consideration, the design of the FRA and MPPM mechanisms is divided into three specific steps that have to be included in the modeling procedure: the first is the design of an appropriate template, which will be illustrated in a small and obvious example in Figure 1; the second step describes the automated modeling by defining and explaining the necessary parts of the module, programmed by the modeler following a certain algorithm as explained in Section 4.6; the third step is the verification, where the modeler checks whether all has been applied as intended.

\subsection{Description of Emergency Department}

The Irish ED of a large academic teaching hospital shares the resources of several departments, such as diagnostic radiology or the laboratory where blood tests are diagnosed. There are three main patient types who are seen in the ED: self-referred walk-in patients, referred patients with a letter from their General Practitioner (GP), and acute patients arriving by ambulance. The ED accepts all patients and provides immediate treatment for almost all kinds of injuries and complaints. Only a few exceptional cases are not treated, such as burns, pediatrics, or mental illnesses. Those patients are transferred to an appropriate hospital. Accepted patients are classified by their severity into triage groups and into complaint groups according to the Manchester Triage Group (Mackway-Jones, Marsden, and Windle 2006). The complaint groups indicate the specific pathway for the patient through the ED. The patients who are treated and require no further follow up treatment are directly sent home. Those that need follow up treatment are either sent to a General Practitioner or are transferred to a different specialty unit within the ward or of a different hospital. Patients are also transferred into the ward directly; the hospital is operating near their capacity limit which results in long waiting queues for the warded patients.

From the perspective of the patient, the ED is an additional healthcare facility where immediate care can be provided even in cases where a GP is not available. In the Irish context, the ED is a supplement to the available healthcare infrastructure. Patients are still treated even though they are not an emergency case, as the name might implicate. Annually, around 45,000 patients visit the ED with approximately 50 different patients groups, which averages to 3,750 patients each month. Patient demand is highest on working days, whereas the demand is at its lowest over the weekend.

The general setting of the ED can be described by following the patient pathway: the treatment is divided into three different treatment areas which are located in three separate units within the ED. The "Chest Pain" Unit which is dedicated to patients who have either a lung or a heart problem. This unit 


\section{Thorwarth, and Arisha}

is equipped similarly to an Intensive Care Unit (ICU) which holds cardiovascular monitoring, provides artificial respiration, syringe drivers and offers infusions. The "Minor Complaints" Unit is dedicated to patients who require orthopedic care and treatment. Such cases are for example fractures, displacements, or wounds that require treatment. The "General Complaints" Unit covers all other patients requiring treatment.

\subsection{Patient Pathway Description}

The most common patient pathway in the ED is displayed as follows: patients must first register before they enter the ED. They are then asked to stay in the waiting room until they are triaged by a triage nurse or doctor. These doctors and nurses are shared resources among the ED and classify the severity of the patient according to the Manchester Triage Group (Mackway-Jones, Marsden, and Windle 2006). Following this, depending on their severity they either continue to wait in the waiting room or are sent directly to one of the three units. After being interviewed, diagnosed and treated in the units, patients are either sent home, referred to a GP or specialty unit of a different hospital (outpatient clinic), or are admitted to the hospital. Patients do die in the ED, but this is not a common occurrence. Potential hospital patients are monitored by the clinical decision unit (CDU) which has the final decision on whether a patient requires hospital treatment or not. The CDU is functionally placed among the ED and the SVUH, even though the patients remain in their units. The main pathway is illustrated in Figure 2 which combines all 50 patient groups together in one flow. A more fully detailed description of the ED can be retrieved from Thorwarth (2011).

\subsection{Data Mining}

Twenty months of hospital records were used to retrieve arrival patterns, patient groups and their corresponding process times such as waiting times and treatment times. Those statistical data are stored according to their complaint group, specialty and referral type in a database that is accessible by the model in order to retrieve the most recent analyzed data.

The workflow, patient pathway and accompanying processes, had been investigated with onsite visits and interviews that involved medical and non-medical staff. In addition, the medical records of ca. 80.000 patients were investigated. The patient records correspond to the duration of twenty months of patient movement through the ED. Those data were grouped and the resulting process parameters obtained are summarized in statistical distributions for each relevant process group.

In order to reduce the number of patient groups and identify the number of relevant groups that are necessary to represent the ED, a correlation analysis is made with the hospital records. The overall average process times are calculated for all patient groups, and hence these values are compared to the average value of the reduced number of groups remaining. The correlation coefficient from the average of all groups to the reduced groups is hence calculated. The number of reduced groups which are below 0.95 marks the value where sufficient groups are available to represent the ED. It has been identified that 14 groups out of 50 patient groups are required for the representation of the ED. The remaining groups are aggregated into one remaining group, which is hence routed through the ED model in the same manner as the relevant statistics from the ED have drawn.

A conceptual model is then developed with the use of the IDEF0 modeling approach and process flow mapping technique. The Figure 2 illustrates the 14 major groups of patients that move through the ED. Internally, the ED is split into three zones: the first group is the Chest Pain Unit, where patients with respiratory and heart complaints are referred to. The second unit is the General Complaints Unit, and the third unit treats minor diseases. Successive departments exist also, like the Clinical Decision Unit which examines the condition of the patient.

\subsection{Conceptual Model}

On site visits, interviews of staff, and hospital records allowed us to draw process flow maps and IDEF0 models that were created in accordance to the management staff of the ED. Time measurements of the 
Thorwarth, and Arisha

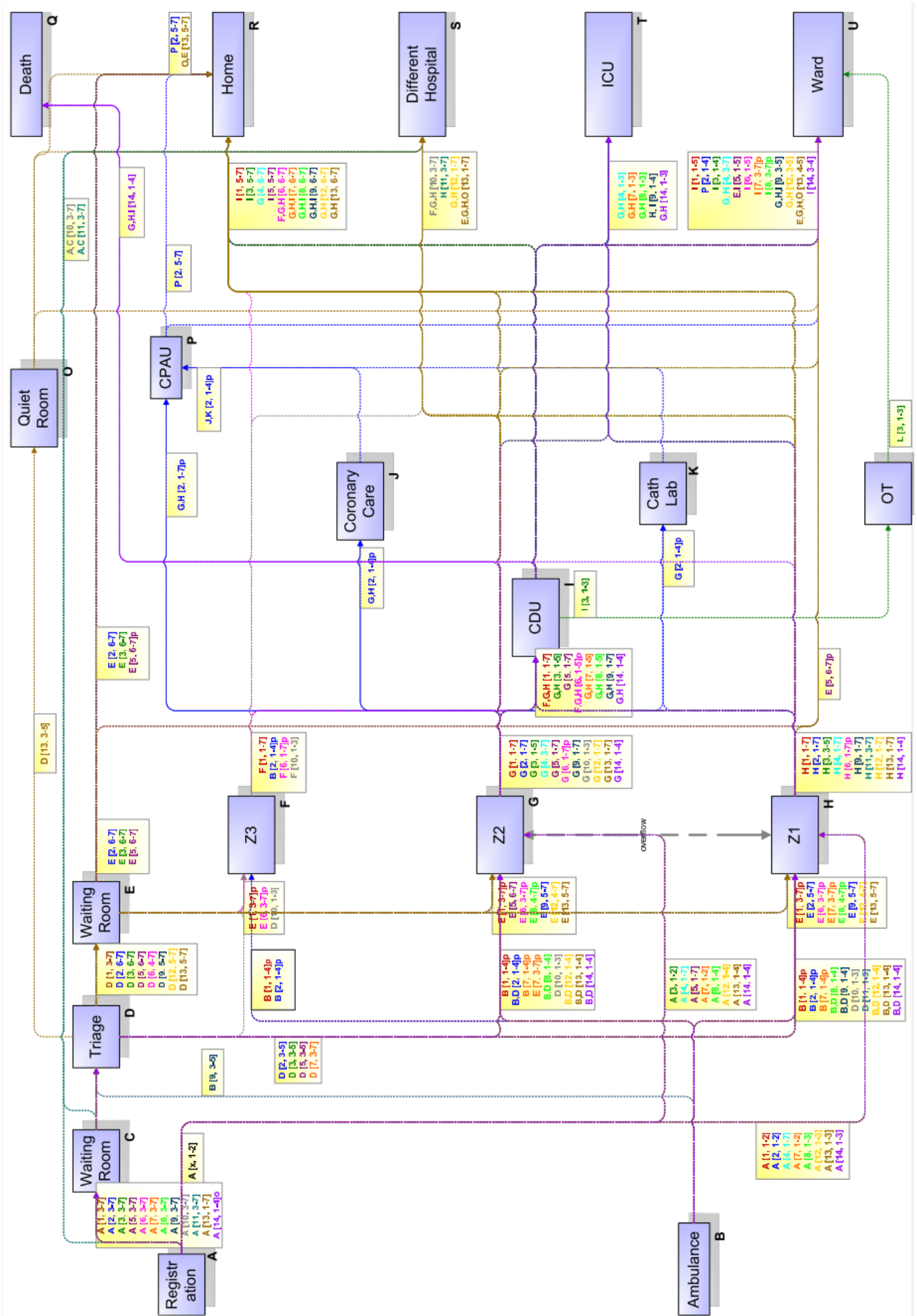

Figure 2: Flow chart of all 14 complaint groups relevant to the ED under investigation. The blue boxes indicate the stations the patient visits during his journey with an attached letter indicating the address of the station. The arrows describe the actual journey, while the yellow boxes contain data about the patient properties: <Previous Station $>$ [ $<$ Complaint Group $>$, $<$ Internal Severity Level $>$ ]. 
Thorwarth, and Arisha

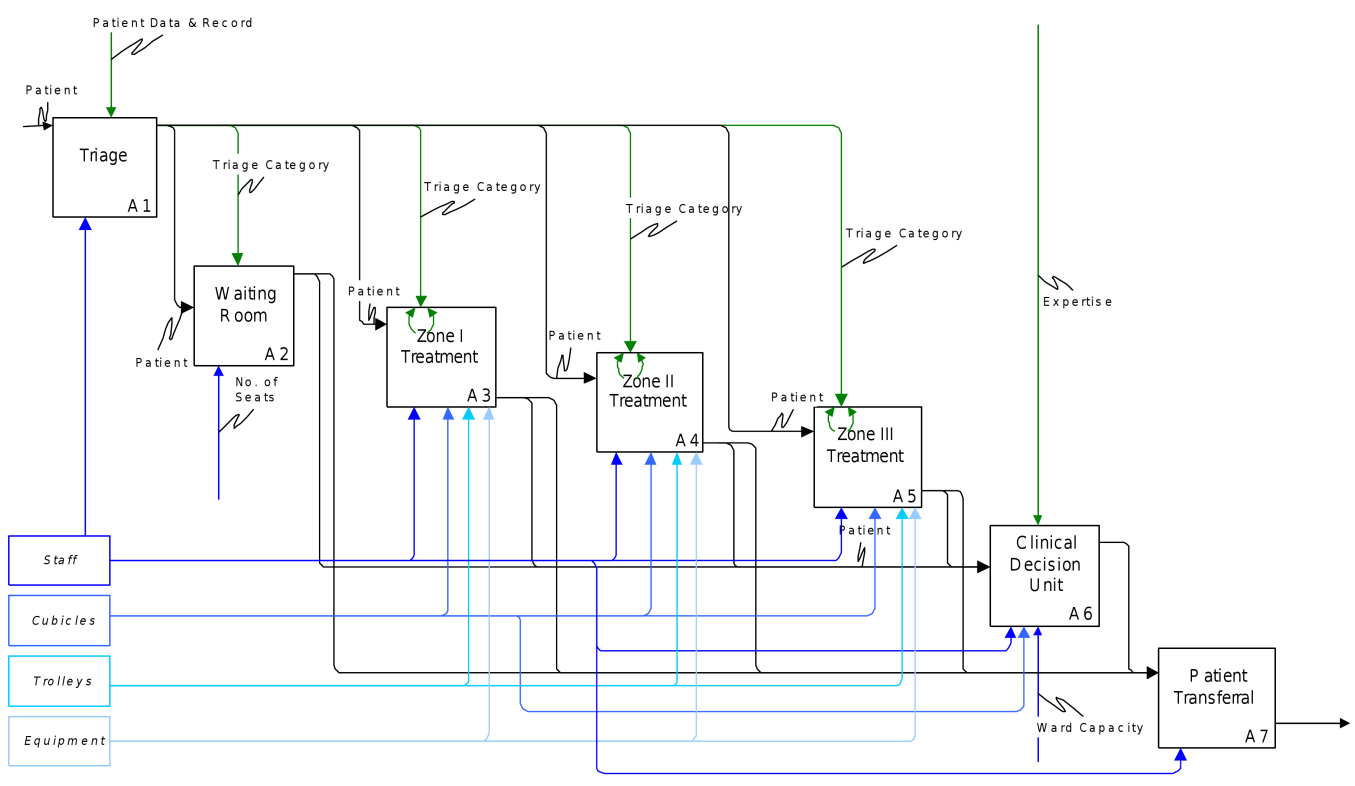

Figure 3: An example of one complaint group (minor injuries) illustrated as an IDEF0-model.

observed processes confirmed the process times retrieved from the hospital records which yield 20 months of patient data. The resulting process map indicates the 14 complaint groups and their corresponding severity status mapped in a process flow diagram, where relevant internal departments and specialties can be obtained (Figure 2). The IDEF0 map illustrates the process steps of the internal processes according to the hierarchy level. An example of the minor injuries complaint group is provided in Figure 3 . The whole IDEF0 model and description of the ED can be found in Thorwarth (2011), whereas a detailed instruction of the application of IDEF0 can be retrieved from National Institute of Standards and Technology (2010). In addition to the illustration of the flow of items through the system, the distribution of resources is mapped within the IDEF0 model. Therefore, a combination of process flow maps and IDEF0 models is an ideal basis for the translation of the conceptual model into an executive model for simulation.

\subsection{Combining Patient Flow with the Workflow of Medical Staff}

During the mapping of the processes of the patient in combination with the workflow of medical staff and resources high attention is demanded from the modeler due to the increased number of pathways that are interlinked with dependent resources. MPPM and FRA can be observed when medical staff, for example a nurse, treats one patient while another doctor is waiting with his treatment for this nurse because he is dependent on his/her assistance. Even though the nurse has completed his/her treatment, there are some final steps which need to be accomplished before she can assist the doctor. Mapping MPPM and FRA thus increases the complexity significantly. To achieve a guided modeling of MPPM and FRA, a framework is proposed which includes an algorithm that allows us to automatically layout a process network grid which can be used as a basic model structure. The adjustment to the final model can therefore be reduced to a minimum. Essential for automated modeling is a runtime environment within the simulation software package that enables controlling of the layout in an automated manner.

\subsection{Automated Modeling}

Automated modeling requires planning and consideration in regard to required resources. This step contributes to planning the layout section and the programming section. The programming section influences the automated modification of the model. The layout is beneficial in most simulation software packages 


\section{Thorwarth, and Arisha}

as it allows a clean layout even on a high complexity degree. This mapping can be allocated by providing mapping instructions; however, every individual simulation software package applies different procedures which can be accessed with their programming interface.

In order to understand how the mechanism of FRA and MPPM are translated into the modeling of the simulation model, the algorithm is illustrated as an example in Figure 4. The creation of the process grid can be followed which is created by the automated modeling module. Interested readers that would like to rebuild their own automated modeling module are referred to Thorwarth (2011), where a detailed instruction for the programming and design of this module is provided.

The illustrated example is divided into three elemental steps of which parameters determine the resulting size of the process grid considering FRA and MPPM. Those parameters describe the length of the longest process chain of the system under investigation, the number of process chains involved, and the number of flexible allocated resources. An important preparation is the definition of the starting process which is designed as a template. In this case (Figure 4) the template is the key starting process of the process chain $A_{1}$. Important to the consideration of FRA and MPPM, is to attach the Sender and Receiver to the key starting process. Their function is to provide an interface to the resources. The process grid can be deflated after having implemented the automated modeling module, the template, and set the parameters. In step 1 the template is copied and connected to the previous process until the number of required elements in the process chain is reached. In step 2 the process chain is duplicated to the essential number required (here $n=2$ ). The process chains describe the passive pathways, which is the patient flow in our example. Step 3 creates the resource groups which include the resource pools and the accompanying processes that describe the active pathway (here workflow of staff). The Sender and Receiver are here required to transfer the resource to the required processes of the passive pathway. Within the last step, all addresses of the Senders and Receivers are initialized so that the resources are moving within this grid in a reliable manner.

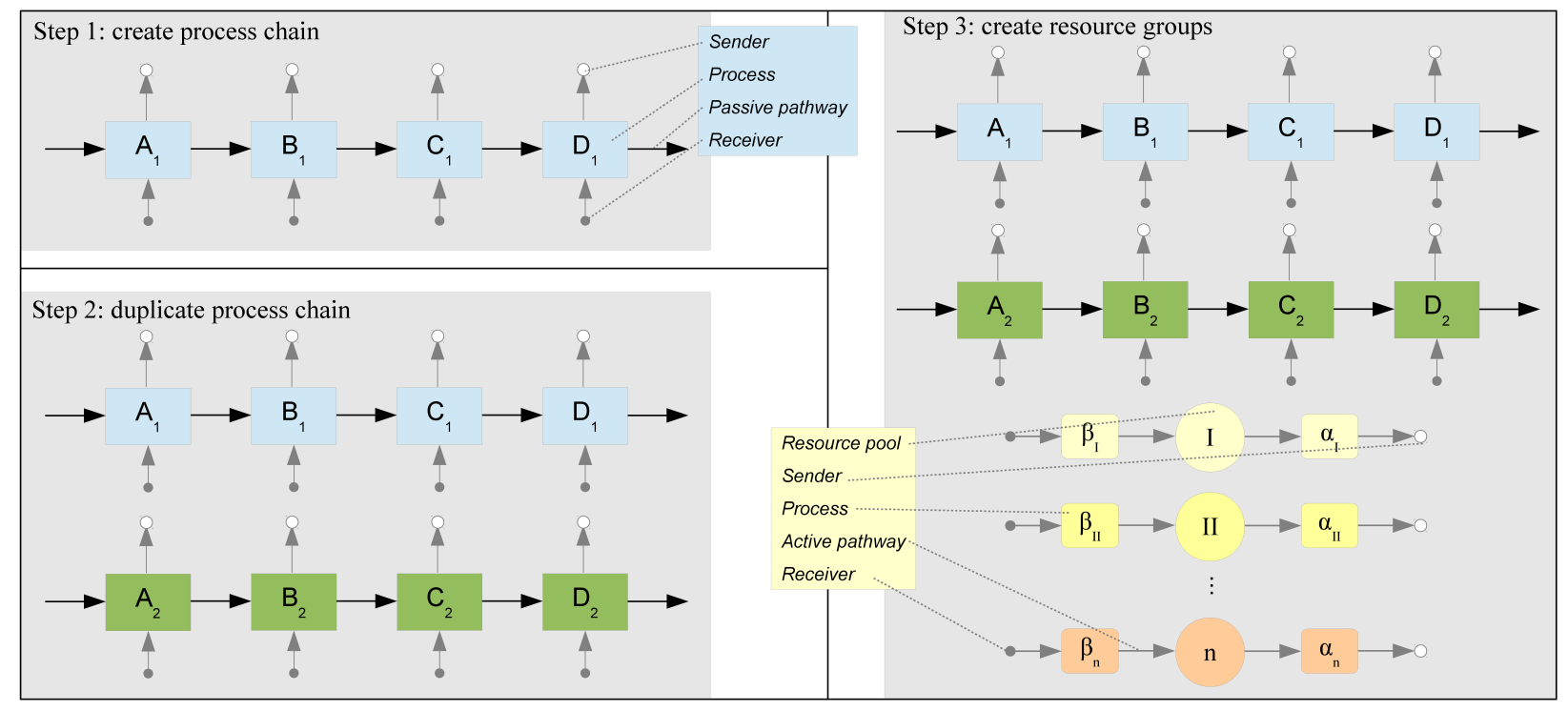

Figure 4: Illustrated algorithm of the automated modeling module considering FRA and MPPM. Step 1: Creation of the process chain with its interfaces to the later created resource groups. Step 2: Duplication of the required number of process chains. Step 3: Creation and connection of the resource groups with the passive pathways.

Within this process grid, the resources are drawn to the process where the resources are required. This mechanism represents the FRA described in Section 3.1, while the presence of the passive and active pathway allow the resources to move within the network as introduced in Section 3.2. 


\section{Thorwarth, and Arisha}

Having extracted the process flow grid which displays the workflow and the patient pathway in a raw state, the modeler has to adjust the raw model to the conceptual model. Adjustments to the routing have to be applied and the process times have to be inserted into the model. Those manual adjustments can be regarded as the clearance of unused process routes, and are therefore easier to maintain than it would be to build up those processes from scratch.

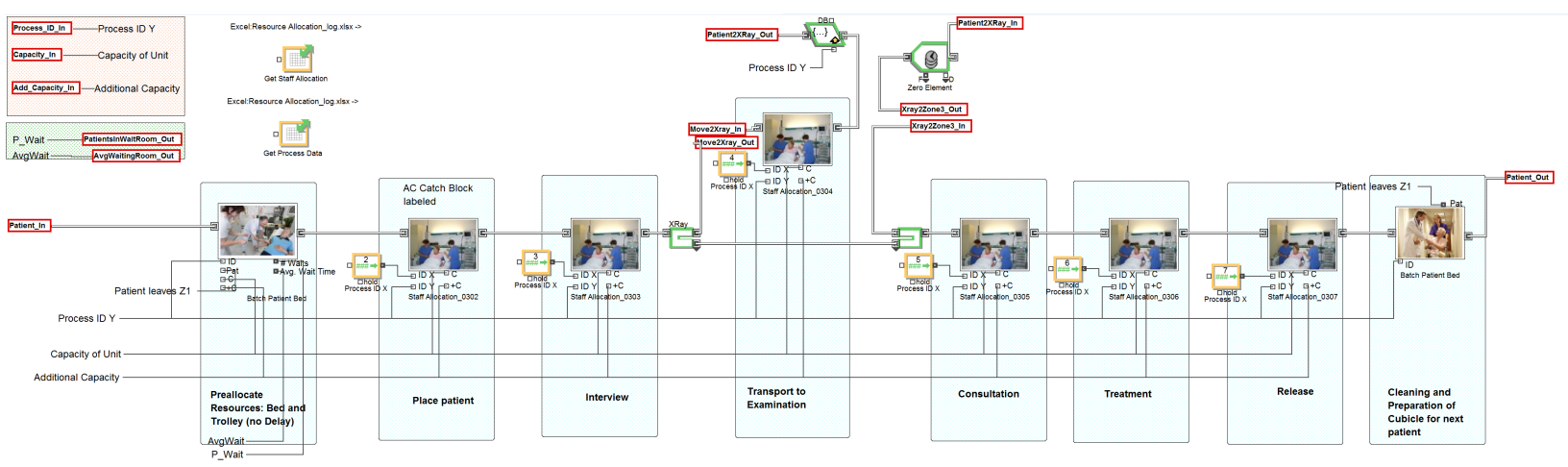

Figure 5: Screenshot of one process chain (passive pathway) applied for one of the three treatment zones within the ED.

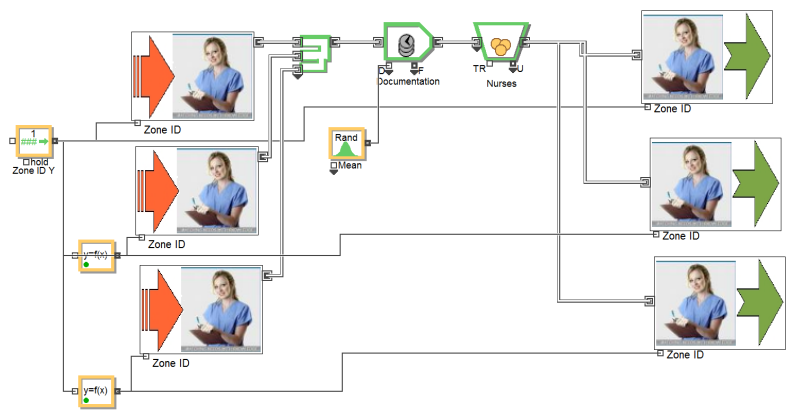

Figure 6: Screenshot of one resource group (active pathway) applied within the ED.

The Figures 5 and 6 provide snapshots of the original model, which has been applied at the ED under investigation. Further details and more information regarding the translation of the automated modeling module can be obtained from Thorwarth (2011).

\subsection{Verification and Validation}

In order to verify the simulation model, hospital records are compared with simulation results. The average number of process steps differs within a 5\% range (best 1.7\%, worst 9.9\%), however one outlier is observed which is a $9.9 \%$ wait within the second wait. This difference is still within the $99 \%$ confidence interval. An additional important observation for the interpretation and verification of results is that the averages of the process times of four of the six main hospital processes are within the $99 \%$ confidence interval. It can therefore be concluded that the probability of the simulation result being within that range is $99 \%$. The box plot in Figure 7 provides a visual example of the verification results by indicating the deviation from the simulation results to the hospital records.

In addition to the verification, it could be seen during the modeling that animation proved exceptionally useful for tracing the elements through the system, especially when the active pathways described by the FRA have to be debugged. 
Thorwarth, and Arisha

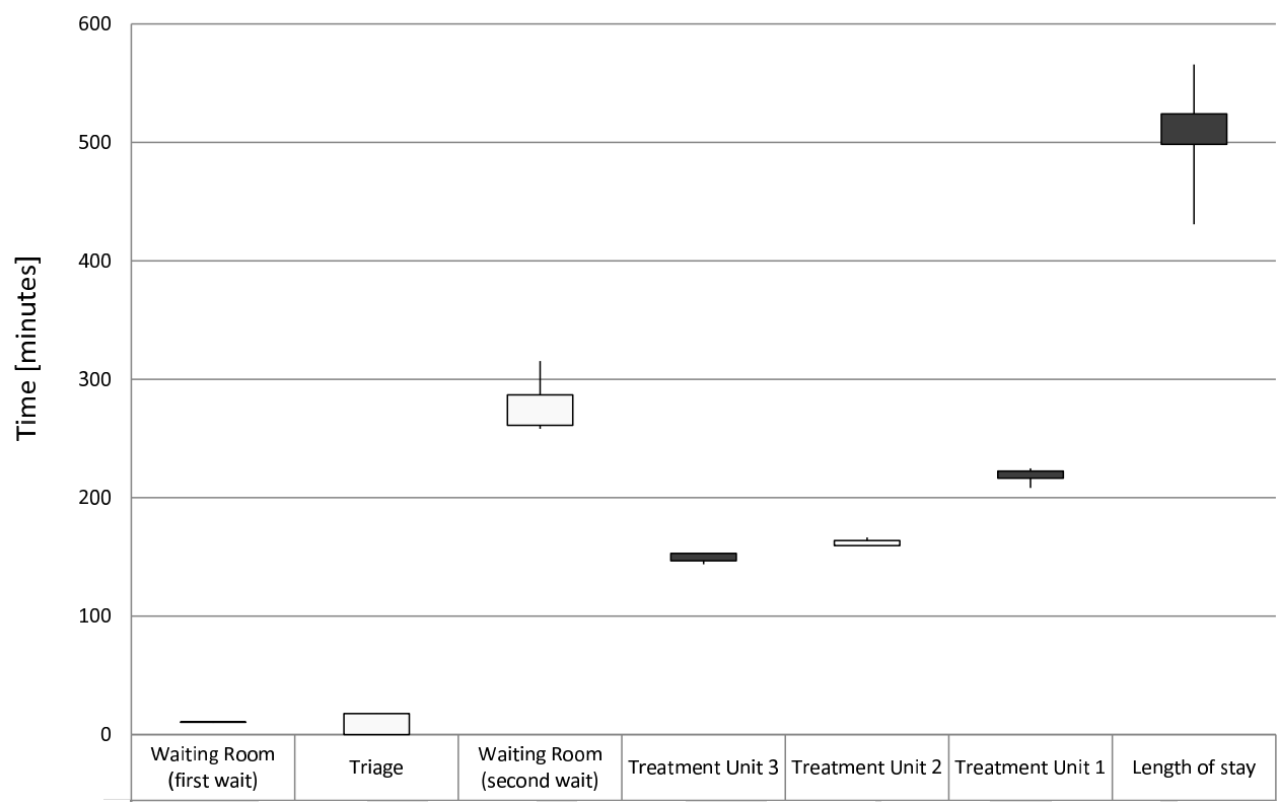

Figure 7: Verification of key process times from the simulation results compared with the hospital records.

The comparison of results for the verification of the correct model translation indicate that the overall process times and the initial identified process inputs are within an acceptable range. Therefore the model can be considered as a representative picture of the processes within the ED under investigation. This model is then used to test scenarios which examine the impact of documentation on the processes and show how queuing time can be reduced by the integration measures reducing documentation time.

\section{RESULTS}

With the aid of a model which simulates MPPM and FRA, policies that are influencing the patient pathways and the workflow of medical staff can be investigated. The introduction of a documentation aid such as speech recognition software or digital pens etc. are generally assumed to impact patient pathways by supporting and optimizing the workflow of staff.

The impact on the patient pathway is measured in LoS, and the impact on the medical staff is measured in turnaround time and utilization. The results show that while staff utilization is below 75 percent, the impact on the patient pathway is not significantly observable. However, it is observed that the reduction of documentation time by just of minute per process step accumulates up to 27 minutes per shift and staff member on average. The relation between the two process types is explained by the fact that a high impact of the documentation effort would have occurred if the utilization would have been close to 100 percent. This would have had the consequence that the staff pool would be empty and that requests for medical staff could not be fulfilled because the staff would have been occupied with documenting or other tasks. As this is not the case and the utilization indicates enough idle resources remaining in the staff pool, the documentation reduction has little impact on the patients' journey through the ED, even though it has a relatively significant impact on the overall workload.

\section{CONCLUSION}

The result of the scenario analysis presented in Section 5 indicates that the introduction of a documentation aid has a minor impact on the LoS of patients. However, benefits can still be expected on the side of 


\section{Thorwarth, and Arisha}

the utilization level of medical staff. The overall workload would be reduced and capacity therefore be increased.

Modeling and simulating both process types, patient pathways and workflow of medical staff provide decision makers with a precise system that examines the impact of both interlinked process flow types. In the case of the documentation aid, it is essential to take both process flow types into account. Just focusing on one type of processes, like the patient pathway, would thus lead to false conclusions.

However, simulating multiple process flows raise the complexity of the model significantly. Large and complex models increase the probability of errors. These errors are difficult to detect due to the size of the models. The frequently given advice to keep simulation models small and simple is unfortunately hard to follow in complex healthcare systems. Framework and guidance for the modeler are essential in order to improve the quality of the developed model. Therefore, the algorithm proposed is a valuable tool for the healthcare modeler as it provides a raw model that can be adjusted to the requirements of the system under investigation. Complex and sophisticated models are thus developed in an easier manner, with less effort required for maintenance and they open a new scope for further development in that area.

Research opportunities are emerging either in different application fields, such as process analysis in laboratories, where analysis of substances have to be done according to certain operational procedures or the expansion / enhancement of a toolkit by integrating staff scheduling into the FRA approach. A good example for staff rostering can be retrieved from Glass and Knight (2010) which would be a worthwhile consideration to be integrated into the staff allocation of this modeling concept.

\section{ACKNOWLEDGMENTS}

The authors would like to express their acknowledgements for our partner hospital in Dublin that facilitated and supported this research with invaluable data, insights into the emergency department, and inspiration to conduct the research. Special thanks to Prof. Dr. John Ryan and Dr. Ian Callanan.

\section{REFERENCES}

Banks, J., J. S. Carson II, B. L. Nelson, and D. M. Nicol. 2005. Discrete-Event System Simulation. 4th ed. Prentice Hall International Series in Industrial and Systems Engineering. London: Pearson Prentice Hall.

Berge, C. 1976. Graphs and hypergraphs. North-Holland Pub. Co.

Eatock, J., R. Paul, and A. Serrano. 2001. "A study of the impact of information technology on business processes using discrete event simulation: a reprise". International Journal of Simulation Systems, Science \& Technology 2:30-40.

Glass, C. A., and R. A. Knight. 2010. "The nurse rostering problem: A critical appraisal of the problem structure". European Journal of Operational Research 202 (2): 379 - 389.

Günal, M. M., and M. Pidd. 2006, December. "Understanding accident and emergency department performance using simulation". In Proceedings of the 2006 Winter Simulation Conference, edited by L. F. Perrone, F. P. Wieland, J. Liu, B. G. Lawson, D. M. Nicol, and R. M. Fujimoto, 446-452. Piscataway, New Jersey: Institute of Electrical and Electronics Engineers, Inc.

Günal, M. M., and M. Pidd. 2010. "Discrete event simulation for performance modelling in health care: a review of the literature". Journal of Simulation 4 (1): 42-51.

Harzing, A.W. 2010. "Publish or Perish, version 2.8". Last accessed on 4th of April 2010.

Hoot, N. R., L. J. LeBlanc, I. Jones, S. R. Levin, C. Zhou, C. S. Gadd, and D. Aronsky. 2008. "Forecasting Emergency Department Crowding: A Discrete Event Simulation". Annals of Emergency Medicine 52 (2): 116-125.

Jun, J. B., S. H. Jacobson, and J. R. Swisher. 1999. "Application of discrete-event simulation in health care clinics: A survey". Journal of the Operational Research Society 50 (2): 109-123. 


\section{Thorwarth, and Arisha}

Lassen, K. B., and W. M. P. van der Aalst. 2009. "Complexity metrics for Workflow nets". Information and Software Technology 51 (3): 610-626.

Mackway-Jones, K., J. Marsden, and J. Windle. 2006. Emergency Triage. 2nd ed. Oxford: Blackwell Publishing.

McCabe, T. J. 1976. "A complexity measure". In IEEE Transactions on Software Engineering, Volume 2, 308-320: IEEE Computer Society Press.

National Institute of Standards and Technology 2010, October. "Integration Definition for Function Modeling (IDEF0)". last accessed on 21th of June 2011.

Rohleder, T., D. Bischak, and L. Baskin. 2007. "Modeling patient service centers with simulation and system dynamics". Health Care Management Science 10:1-12.

Ruohonen, T., P. Neittaanmäki, and J. Teittinen. 2006, December. "Simulation model for improving the operation of the emergency department of special health care". In Proceedings of the 2006 Winter Simulation Conference, edited by L. F. Perrone, F. P. Wieland, J. Liu, B. G. Lawson, D. M. Nicol, and R. M. Fujimoto, 453-458. Piscataway, New Jersey: Institute of Electrical and Electronics Engineers, Inc.

Thorwarth, M. 2011. A Simulation-based Decision Support System to Improve Healthcare Facilities Performance elaborated on an Irish Emergency Department. Ph. D. thesis, Dublin Institute of Technology.

Thorwarth, M., P. Harper, and A. Arisha. 2009, December. "Simulation model to investigate flexible workload management for healthcare and servicescape environment.". In Proceedings of the 2009 Winter Simulation Conference, edited by M. D. Rossetti, R. R. Hill, B. Johansson, A. Dunkin, and R. G. Ingalls, 1946-1956. Piscataway, New Jersey: Institute of Electrical and Electronics Engineers, Inc.

Wild, P. J., P. Johnson, and H. Johnson. 2004, November 15-16 2004. "Towards a composite modelling approach for multitasking". In 3rd Annual Conference on Task Models and Diagrams (TAMODIA 2004), 17-24. Prague, Czech Republic: ACM Press.

Yeh, J.-Y., and W.-S. Lin. 2007. "Using simulation technique and genetic algorithm to improve the quality care of a hospital emergency department". Expert Systems with Applications 32 (4): 1073-1083.

\section{AUTHOR BIOGRAPHIES}

MICHAEL THORWARTH, Ph.D. is a Lean / Six Sigma Specialist at Baxter Oncology GmbH and acting as an academic advisor for the $3 \mathrm{~S}$ Group (Research unit in D.I.T which specializes in complex systems simulation and optimization). He has recently completed his PhD in Process Management at the Dublin Institute of Technology, Ireland. He is a Diplom-Ingenieur (German equivalent to M.Eng.) in Medical Computing and Biomedical Engineering which he studied at the Technical University of Ilmenau, Germany. His research interests focus on the application of discrete event simulation, process management and optimization in healthcare and process management. His email address is michael.thorwarth@gmail.com.

AMR ARISHA, Ph.D. is the director of the $3 \mathrm{~S}$ Group and the head of international business department in college of business in Dublin Institute of Technology. He Joined DIT in 2005, he received his PhD Industrial Engineering from Dublin City University (DCU). Intel-Ireland has sponsored his research from 2000 - 2005. Dr. Arisha has published many journal and international conference articles in the area of manufacturing systems simulation and scheduling. His research interests include analysis and optimization of complex dynamic systems in manufacturing and service industries using stochastic simulation modeling, and optimization techniques. His research also includes Supply Chain Management, Operations Excellence, Project Management, Virtual Reality applications in Business Process Analysis, and Optimization for Process Improvement. He is a member in IIE, IMECH, IEI, ESE, ORS, IEEE and ASME. His email address is amr.arisha@dit.ie. 